Acta Cryst. (1989). C45, 1411-1412

\title{
Manganese(II) Hydrogenphosphate Trihydrate
}

\author{
By Y. Cudennec, A. Riou et Y. Gérault \\ Laboratoire de Chimie des Matériaux Inorganiques et de Cristallographie, Institut National des \\ Sciences Appliquées, 20 avenue des Buttes de Coesmes, 35043 Rennes CEDEX, France
}

(Received 8 February 1989; accepted 15 March 1989)

https://doi.org/10.1107/S0108270189003604

\begin{abstract}
Mn}\left[\mathrm{PO}_{3}(\mathrm{OH})\right] \cdot 3 \mathrm{H}_{2} \mathrm{O}, \mathrm{M}_{\mathrm{r}}=204.96$, orthorhombic, Pbca, a $=10.408(3), \mathrm{b}=10.863(3), \mathrm{c}$ $=10.192$ (4) $\AA, \mathrm{V}=1152.3(1.1) \AA^{3}, \mathrm{D}_{\mathrm{x}}=2.353$ (3) Mg.m ${ }^{-3}, \mathrm{Z}=8$, Mo $K \alpha, \lambda=0.71073 \AA, \mu=$ $2.440 \mathrm{~mm}^{-1}, \mathrm{~F}(000)=824$, room temperature, $\mathrm{R}=0.022$ for 1120 observed reflections. The structure is isotypic with newberyite: $\mathrm{Mg}\left[\mathrm{PO}_{3}(\mathrm{OH})\right] \cdot 3 \mathrm{H}_{2} \mathrm{O}$.
\end{abstract}

Experimental. Crystals of $\mathrm{Mn}\left[\mathrm{PO}_{3}(\mathrm{OH})\right] \cdot 3 \mathrm{H}_{2} \mathrm{O}$ were obtained from a mixture of manganese carbonate, phosphoric acid and water. After elimination of carbon dioxide, the reacting system belongs to the ternary system $\mathrm{MnO}, \mathrm{P}_{2} \mathrm{O}_{5}, \mathrm{H}_{2} \mathrm{O}$. A mixture containing $8 \% \mathrm{MnO}, 12 \% \mathrm{P}_{2} \mathrm{O}_{5}, 82 \%$ $\mathrm{H}_{2} \mathrm{O}$ (in weight) was slowly dehydrated at room temperature, without stirring. Colourless flattened octahedral crystals appeared, of average size $0.2 \mathrm{~mm}$. A suitable crystal $(0.2 \times 0.2 \times 0.3 \mathrm{~mm})$ was mounted on an Enraf Nonius CAD-4 diffractometer using Mo $K \alpha$ radiation. The unit-cell constants were determined and refined on the basis of 25 carefully centred reflections in the angular range $0<$ $2 \theta<30^{\circ}$. Data were collected in the $\omega-2 \theta$ scan mode with $\omega$-scan width $(2.00+0.50 \operatorname{tg} \theta)^{\circ}$ and slit amplitude $(1.00+0.35 \operatorname{tg} \theta) \mathrm{mm}$. A total of 1621 reflections were measured. The $h, k, l$ range was $0 \leq$ $h \leq 13, \quad 0 \leq k \leq 14, \quad 0 \leq l \leq 13\left(2 \theta<56^{\circ}\right)$. A periodic check of three standard reflections showed no significant intensity variation. Reflections were merged to a set of 1385 . According to $I>\sigma(I)$, 1120 reflections were regarded as observed. Data were corrected for Lorentz and polarization effects and absorption corrections were applied. The structural determination was carried out on a PDP 11/60 computer using the SDP package (Frenz, 1978). Scattering factors were from Cromer \& Waber (1965). The structure has been studied on the basis of isomorphism with $\mathrm{Mg}\left[\mathrm{PO}_{3}(\mathrm{OH})\right] \cdot 3 \mathrm{H}_{2} \mathrm{O}$, whose structure was determined by Sutor (1967), and refined with the $\mathrm{H}$ atoms by Abbona, Boistelle \& Haser (1979). Refinement was started with atomic parameters reported by Abbona et al. (1979). The seven H atoms were refined isotropically. Full-matrix leastsquares refinement was based on $\mathrm{F}$ and the function minimized was $\sum w\left(\left|F_{0}\right|-\left|F_{c}\right|\right)^{2}$, where $w(F)$ $=1 / \sigma^{2}(F)$. The weighting scheme had a non-Poisson contribution with $p=0.050$. Corrections were made for anomalous dispersion and secondary-extinction effects $\left(g=1.29 \times 10^{-7}\right)$. During the last refinement cycle, the r.m.s. shift/e.s.d. was 0.07 . The final difference Fourier map showed no features higher than $0.39 \mathrm{e} \AA^{-3}$. In these conditions, the final $R$ was 0.022 and $w R=0.033$.

Final atomic coordinates and equivalent isotropic temperature factors are given in Table l.* 
Related literature. The results of a study of divalent manganese phosphates are reported in a recent paper (Cudennec, Riou \& Gerault, 1986). Several well crystallized phases were prepared, mainly manganese hydrogenphosphates. Little is known about such solid-phase structures. Recently we have published the structural determination of a new manganese phosphate hydrogenphosphate: $\mathrm{Mn}_{7}\left(\mathrm{PO}_{4}\right)_{2}\left[\mathrm{PO}_{3}(\mathrm{OH})\right]_{4}$ (Riou, Cudennec \& Gerault, 1987), and refined the structure of synthetic hureaulite of pure manganese: $\mathrm{Mn}_{5}\left(\mathrm{PO}_{4}\right)_{2}\left[\mathrm{PO}_{3}(\mathrm{OH})\right]_{2} \cdot 4 \mathrm{H}_{2} \mathrm{O}$ (Gerault, Riou \& Cudennec, 1987). The isomorphism of $\mathrm{Mn}\left[\mathrm{PO}_{3}(\mathrm{OH})\right] \cdot 3 \mathrm{H}_{2} \mathrm{O}$ with $\mathrm{Mg}\left[\mathrm{PO}_{3}(\mathrm{OH})\right] \cdot 3 \mathrm{H}_{2} \mathrm{O}$ (newberyite) was first established by Durif (1971).

* Lists of structure factors and anisotropic thermal parameters have been deposited with the British Library Document Supply Centre as Supplementary Publication No. SUP 51833 (9 pp.).

Copies may be obtained through The Executive Secretary, International Union of Crystallography, 5 Abbey Square, Chester CH12HU, England.

\section{References}

Abbona, F., Boistelle, R. \& Haser, R. (1979). Acta Cryst. B35, 2514-2518.

Cromer, D. T. \& Waber, J. T. (1965). Acta Cryst. 18, 104-109.

Cudennec, Y., Riou, A. \& Gerault, Y. (1986). C. R. Acad. Sci. Ser. II, 302, 1149-1154.

Durif, A. (1971). Bull. Soc. Fr. Minéral. Cristallogr. 94, 556-557.

Frenz, B. A. (1978). The Enraf-Nonius CAD-4 SDP - A RealTime System for Concurrent X-ray Data Collection and Crystal Structure Solution. In Computing in Crystallography, edited by H. Schenk, R. Olthof-HazeKamp, H. van Koningsveld \& G. C. BASSI, pp. 64-71. Delft Univ. Press.

Gerault, Y., Riou, A. \& Cudennec, Y. (1987). Acta Cryst. C43, $1829-1830$.

Riou, A., Cudennec, Y. \& Gerault, Y. (1987). Acta Cryst. C43, 821-823.

Sutor, D. J. (1967). Acta Cryst. 23, 418-422. 
Table 1. Final atomic coordinates and equivalent isotropic temperature factors for $\mathrm{Mn}\left[\mathrm{PO}_{3}(\mathrm{OH})\right] .3 \mathrm{H}_{2} \mathrm{O}$

$$
B_{\text {eq }}=\frac{4}{3} \sum_{i} \sum_{j} \beta_{i j} \mathbf{a}_{i} \mathbf{a}_{j}
$$

\begin{tabular}{|c|c|c|c|c|}
\hline & $x$ & $y$ & $z$ & $B_{\text {eq }}\left(\AA^{2}\right)$ \\
\hline $\mathrm{Mn}$ & $0 \cdot 29919$ & $0 \cdot 24726$ & $0.08836(3)$ & $0.953(5)$ \\
\hline $\mathrm{P}$ & $-0.00753(5)$ & $0 \cdot 13224(4)$ & $0 \cdot 15078(5)$ & $0.797(8)$ \\
\hline $\mathrm{O} 1$ & $-0.0872(2)$ & $0 \cdot 2018$ & $0 \cdot 2480(1)$ & $1.60(3)$ \\
\hline $\mathrm{O} 2$ & $-0.0433(1)$ & $0 \cdot 1595$ (1) & 0.0077 (1) & $1 \cdot 21(3)$ \\
\hline $\mathrm{O} 3$ & $0 \cdot 1371(1)$ & $0 \cdot 1500(1)$ & $0 \cdot 1698$ (1) & $1 \cdot 23(2)$ \\
\hline O4 & $-0.0356(1)$ & -0.0098 & $0 \cdot 1766(2)$ & $1 \cdot 39$ (3) \\
\hline $\mathrm{O} 5(w)$ & $0 \cdot 1637(2)$ & $0 \cdot 3186(2)$ & $-0.0625(2)$ & $1.91(3)$ \\
\hline O6(w) & $0 \cdot 2495(2)$ & $0 \cdot 4195$ (1) & $0 \cdot 1929(2)$ & $1 \cdot 88(3)$ \\
\hline $\mathrm{O} 7(w)$ & $0 \cdot 3610(2)$ & $0 \cdot 0906(1)$ & $-0.0354(2)$ & $2 \cdot 50(4)$ \\
\hline $\mathrm{H} 1(\mathrm{O} 5)$ & $0 \cdot 169(4)$ & $0.324(3)$ & $-0 \cdot 140$ & $3 \cdot 0^{*}$ \\
\hline $\mathrm{H} 2(\mathrm{O} 5)$ & $0 \cdot 107(4)$ & $0 \cdot 294(3)$ & -0.055 & $3 \cdot 0^{*}$ \\
\hline H3(O6) & $0 \cdot 290(3)$ & $0.487(4)$ & $0 \cdot 181$ (4) & $3 \cdot 0^{*}$ \\
\hline $\mathrm{H} 4(\mathrm{O} 6)$ & $0 \cdot 187(3)$ & $0.435(3)$ & $0 \cdot 226(4)$ & $3 \cdot 0^{*}$ \\
\hline $\mathrm{H} 5(\mathrm{O} 7)$ & $0 \cdot 370(4)$ & $0 \cdot 112(3)$ & $-0 \cdot 107(3)$ & $3 \cdot 0^{*}$ \\
\hline $\mathrm{H} 6(\mathrm{O} 7)$ & $0 \cdot 352(4)$ & $0 \cdot 018(3)$ & -0.029 & $3 \cdot 0^{*}$ \\
\hline $\mathrm{H} 7(\mathrm{O} 4)$ & -0.015 & $-0.047(3)$ & $0 \cdot 113(4)$ & $3 \cdot 0^{*}$ \\
\hline
\end{tabular}

* Atoms refined isotropically. 